Proceedings

\title{
Development of Force Plates Installed with Five-Axis Load Cells and Analysis Using a Wearable Sensor System ${ }^{\dagger}$
}

\author{
Kiyoshi Hirose ${ }^{1,2, *}$ and Akiko Kondo ${ }^{1}$ \\ 1 Tec Gihan Co. Ltd., Kyoto 611-0033, Japan; a.kondo@tecgihan.co.jp \\ 2 Graduate School of Science and Engineering, Doshisha University, Kyoto 610-0321, Japan \\ * Correspondence: kiyoshi.hirose@tecgihan.co.jp; Tel.: +81-774-48-2334 \\ + Presented at the 13th conference of the International Sports Engineering Association, Online, \\ 22-26 June 2020.
}

Published: 15 June 2020

\begin{abstract}
In this study, we developed a new force plate system. Conventional force plates output force and moment by using the signals of three-axis load cells. The developed force plate installs four 5-axis load cells, and each load cell can output 3-axis force and 2-axis moment relating to deflection. The moment components of load cells are used for the compensation of cross talk and the calculation of moment in the force plate. The force plate can measure force and moment on the outside of load cells installed in the force plate. Gait measurement and analysis were conducted by using the developed force plate, a conventional force plate, and a wearable sensor system (wearable force plate). The results of the comparison of the developed force plates and the conventional force plate outputs indicated a difference in performance on the outside of load cells.
\end{abstract}

Keywords: force; force plate; load cell; wearable sensor

\section{Introduction}

A force plate system is used for the measurement of a ground reaction force. The force plate of this system outputs 3-axis force and 3-axis moment components. The force plate consists of four load cells, and the force and moment are calculated by the load cell signals [1]. The force components are calculated by the sum of load cell signals, and the moment components are calculated by the sum and difference of load cells signals. This calculation is conducted based on the Newton-Euler method [2], and the calculations do not consider the moment generated on load cells. The force plate system consists of a base plate, load cells, an amplifier circuit, and a top plate. Previous force plate systems have been installed with four 3-axis load cells. Therefore, an effect of torsion in the top plate generated by an external force caused the measurement error in the previous force plate system. The top plate in the force plate system is thick and heavy to ensure stiffness. The force plate system consists of a calculation function for compensating for errors in the load cells signal and the effect between the plates and load cells. However, the force and moment error are generated by the torsion of the top plate. Furthermore, previous compensation methods cannot compensate force and moment generated on the outside of load cells.

The effect of torsion of the top plate involves a moment generated on the load cells. Hence, we can develop a high-performance force plate system by developing load cells that can measure the moment about the torsion of a top plate, and the load cells are installed to the force plate system. In this study, we developed a new force plate system consisting of 5-axis load cells. The load cells can measure a 3-axis force and a 2-axis moment. The moment indicates the component about the torsion 
of a top plate. Furthermore, we indicate the performance and effectiveness of the developed force plate through measurement and analysis using a wearable sensor system.

\section{Force Plate System}

The developed force plate system is shown in Figure 1. This system installs with 5-axis load cells, a load cell output, a 3-axis force $\left(f_{x}, f_{y}, f_{z}\right)$, and a 2-axis moment $\left(m_{x}, m_{y}\right)$. The force plate signals are calculated by the load cells signals. The size of the force plate is $400 \mathrm{~mm} \times 600 \mathrm{~mm} \times 60 \mathrm{~mm}$. The weight is $22 \mathrm{~kg}$. The rated output is $F_{x}: \pm 2000 \mathrm{~N}, F_{y}: \pm 2000 \mathrm{~N}, F_{z}: 6000 \mathrm{~N}, M_{x}: \pm 1200 \mathrm{~N} \cdot \mathrm{m}, M_{y}: \pm 900 \mathrm{~N} \cdot \mathrm{m}$, $M_{z}: \pm 400 \mathrm{~N} \cdot \mathrm{m}$.

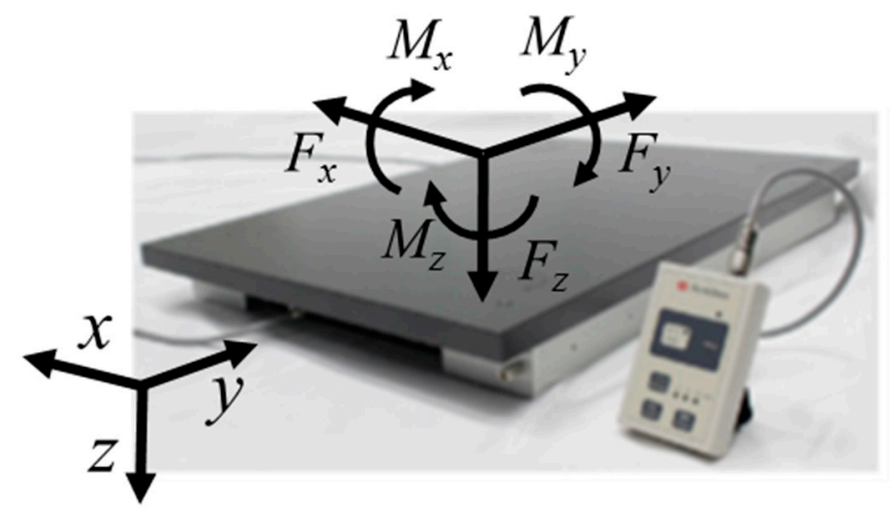

Figure 1. Force plate installed with the 5-axis load cells and definition of strain values about force and moment measured by load cells and the definition of global coordinate system and force plate signals.

Calculation of force and moment is conducted based on the Newton-Euler method [1]. The calculation of force is shown in Equation (1), and the calculation of moment is shown in Equation (2), where $M$ is the moment vector, $f_{i}$ is the force signal outputted from the load cell, and ${ }^{0} p_{i}$ is the position vector from the original point to the load cell [3].

$$
\begin{gathered}
F=\sum_{i=1}^{n} f_{i} \\
F=\left[\begin{array}{l}
F_{x} \\
F_{y} \\
F_{z}
\end{array}\right], f_{i}=\left[\begin{array}{l}
f_{i x} \\
f_{i y} \\
f_{i z}
\end{array}\right] \\
M=\sum_{i=1}^{n}\left({ }^{0} p_{i} \times f_{i}\right) \\
M=\left[\begin{array}{l}
M_{x} \\
M_{y} \\
M_{z}
\end{array}\right],{ }^{0} p_{i}=\left[\begin{array}{l}
{ }^{0} p_{i x} \\
{ }^{0} p_{i y} \\
{ }^{0} p_{i z}
\end{array}\right], f_{i}=\left[\begin{array}{l}
f_{i x} \\
f_{i y} \\
f_{i z}
\end{array}\right]
\end{gathered}
$$

The force plate signals include the error of the load cells signal and the error caused by the attachment of the plate and loadcells. These errors are compensated by the cross-talk compensation matrix. The matrix is generated by the optimal calculation and the calibration device, and these calculations can be combined in the calculation of force and moment. The calculation matrix is shown in Equation (3), where $F_{x, \text { comp }} \sim M_{z, \text { comp }}$ are the compensation values of force and moment. $N_{11} \sim N_{66}$ are the cross-talk compensation elements. The elements are obtained by using the optimal calculation, force plate signal, and the calibration device signal. 


$$
\left[\begin{array}{c}
F_{x, \text { comp }} \\
F_{y, \text { comp }} \\
F_{z, \text { comp }} \\
M_{x, \text { comp }} \\
M_{y, \text { comp }} \\
M_{z, \text { comp }}
\end{array}\right]=\left[\begin{array}{cccc}
N_{11} & \ldots & \ldots & N_{16} \\
& & & \\
\vdots & \ddots & & \vdots \\
\vdots & & \ddots & \vdots \\
& & & \\
N_{61} & \ldots & \ldots & N_{66}
\end{array}\right]\left[\begin{array}{c}
F_{x} \\
F_{y} \\
F_{z} \\
M_{x} \\
M_{y} \\
M_{z}
\end{array}\right]
$$

In previous force plates, the number of load cell signals (strain values) is twelve or eight. The load cell signals are translated by using full bridge circuit $\left(\varepsilon f_{z}\right)$ or half bridge circuit by two load cell signals $\left(\varepsilon f_{x}, \varepsilon f_{y}, \varepsilon m_{x}, \varepsilon m_{y}\right)$ in Wheatstone Bridge circuits, where $\varepsilon f_{x 1} \sim \varepsilon m_{y 2}$ are the load cell signals (strain values). In the force plate system installed with the 5 -axis load cells, the signals number is twelve by including a half bridge circuit in shearing force $\left(\varepsilon f_{x}, \varepsilon f_{y}\right)$ and moment $\left(\varepsilon m_{x}, \varepsilon m_{y}\right)$. The load cell outputs are translated to the force plate outputs by using the translational matrix extended Equation (3). The matrix is shown in Equation (4), $C_{j, k}$ are the elements that combine both a matrix for cross-talk compensation (Equation (3)) and a calculation matrix of force and moment (Equations (1) and (2)).

$$
\left[\begin{array}{c}
F_{x, \text { comp }} \\
F_{y, \text { comp }} \\
F_{z, \text { comp }} \\
M_{x, \text { comp }} \\
M_{y, \text { comp }} \\
M_{z, \text { comp }}
\end{array}\right]=\left[\begin{array}{cccc}
C_{1,1} & \ldots & \ldots & C_{1,12} \\
& & & \\
\vdots & \ddots & & \vdots \\
\vdots & & \ddots & \vdots \\
& & & \\
C_{6,1} & \ldots & \ldots & C_{6,12}
\end{array}\right]\left[\begin{array}{c}
\varepsilon f_{x 1} \\
\vdots \\
\vdots \\
\varepsilon m_{y 2}
\end{array}\right]
$$

The matrix can be estimated by using the optimal value estimation method and the calibration device. The Extended Kalman filter was used for estimating the transformation matrix, and the special calibration device for force plates was used for calibrating the previously developed force plate (TF-4060, TF-6090, TF-90100, Tec Gihan Co. Ltd., Kyoto, Japan). The compensation matrix was calculated by using the calibration device, and the matrix was installed to a special amplifier circuit.

\section{Verification Experiment}

We conducted a verification experiment to confirm the accuracy of the force plate system. The two standard types of force plates (TF-4060, Tec Gihan Co. Ltd.) and two developed force plates were installed in a straight line. The experimental view is shown in Figure 2.

In the experiment, forces were generated between the force plates by stepping between the force plates. The force was measured from a gait motion by using a wearable sensor system (wearable force plate) (M3D force plate, Tec Gihan Co. Ltd.) for the verification [3]. The wearable force plate installs an inertial sensor. The 3D posture (Roll and Pitch angles) in global coordinates is estimated by the inertial sensor signals and the Kalman filter [4], and the force and moment signals in the wearable force plate (sensor coordinate system) were translated to a global coordinate system. The wearable force plate is shown in Figure 3. The total force in force plates and the wearable force plate signals were compared. The experimental view is shown in Figure 4. The sampling frequency is $1000 \mathrm{~Hz}$, and the measurement time is $10 \mathrm{~s}$. 


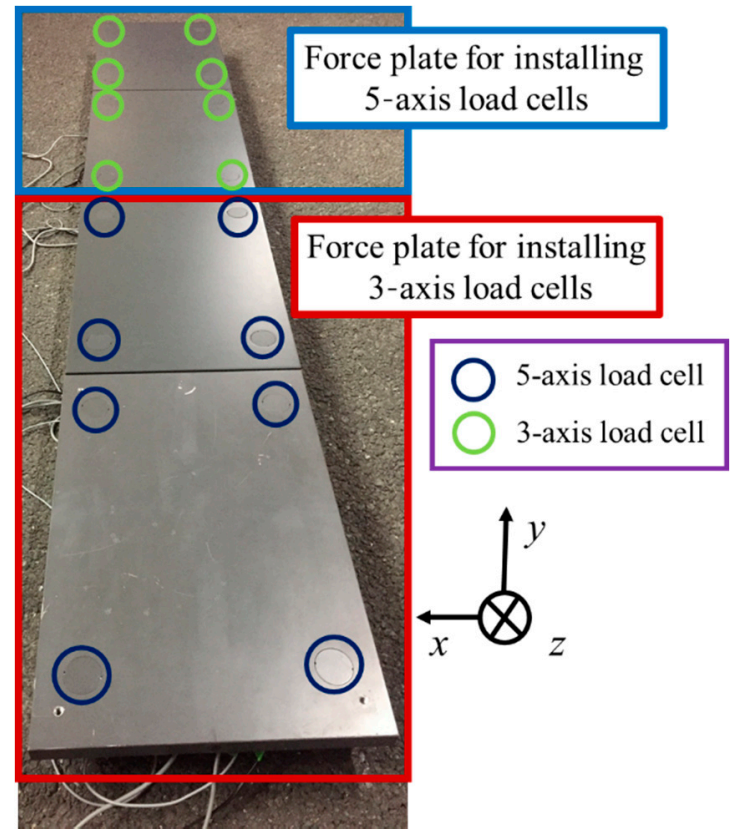

Figure 2. Experimental view and the position of load cells in force plates. The force plates were installed in a straight line, and the height between force plates were adjusted by using a special adjuster by screw bolt mechanism.

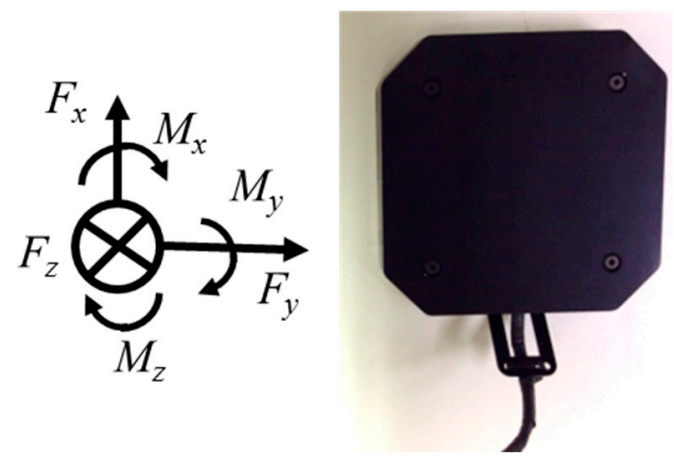

Figure 3. The wearable force plate system consists of four 3-axis compact force sensors. The force plate output 3-axis force and 3-axis moment.

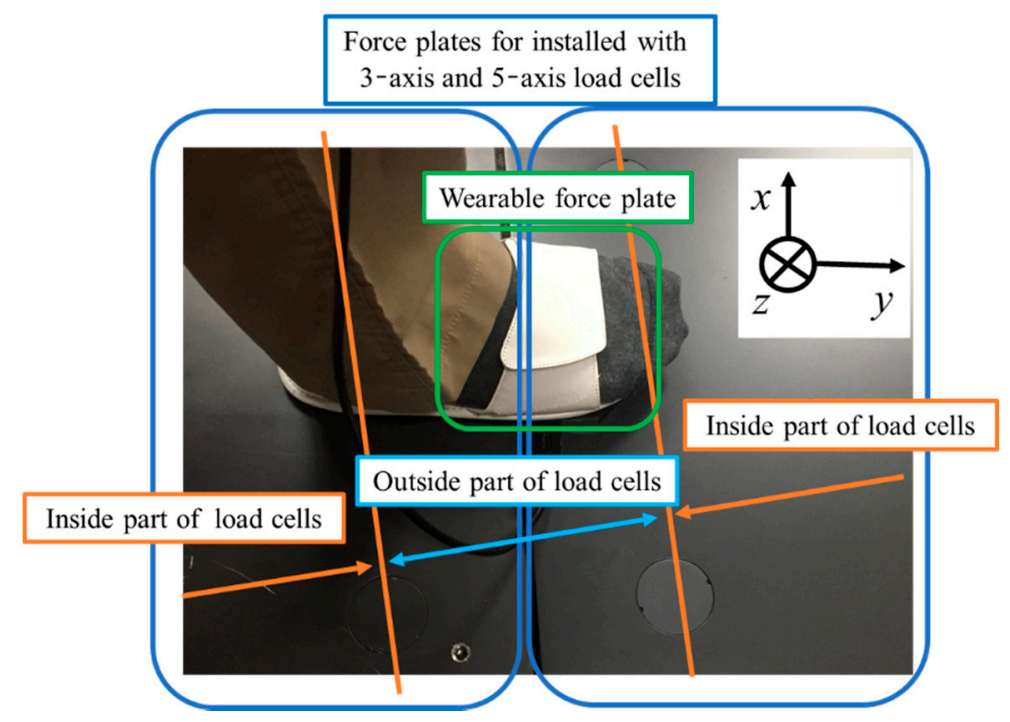

Figure 4. The view of experiment using the force plates installed with 3-axis and 5-axis load cells and the wearable force plate. In this experiment, the force is applied between the two force plates. 


\section{Experimental Results}

Results for the 3-axis force components obtained by the force plates installed with 3-axis and 5axis load cells and the wearable force plate are shown in Figures 5 and 6 . In the results of the force plate installed with 3-axis load cells and the wearable force plate, the $F_{z}$ (vertical direction) component results are in agreement. However, errors were generated in the results of $F_{x}$ (lateral direction) component.

In the results for the force plate installed with 5-axis load cells and the wearable force plate, all component results were in agreement. The root mean square errors were calculated in between the force plates and the wearable force plate. In the force plate installed with 3-axis load cells and the wearable force plate, the root mean square errors were $F_{x}: 9.64 \mathrm{~N}, F_{y}: 9.98 \mathrm{~N}, F_{z}: 10.29 \mathrm{~N}$. In the force plate installed with 5-axis load cells and the wearable force plate, the root mean square errors were $F_{x}: 6.43 \mathrm{~N}, F_{y}: 6.24 \mathrm{~N}, F_{z}: 8.16 \mathrm{~N}$. The error of $F_{x}$ is bigger than other results, and the max value of error in near peak (0.8-1.2 s) are $14.24 \mathrm{~N}$ in the force plate installed with 3-axis load cells and $6.17 \mathrm{~N}$ in the force plate installed with 5-axis load cells. Therefore, the results indicated the effectiveness of force plate installed with 5-axis load cells.

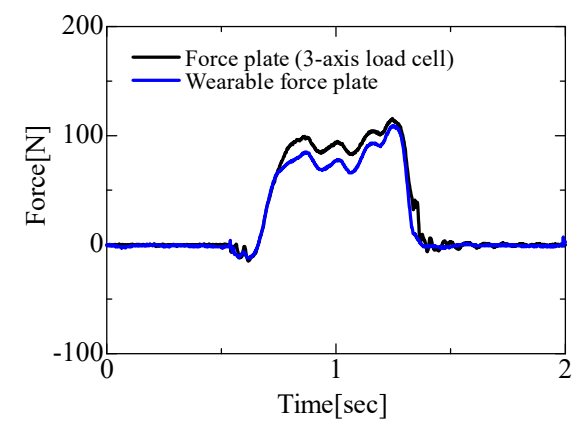

(a)

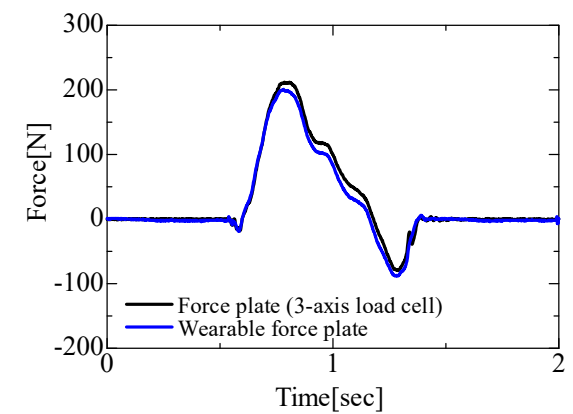

(b)

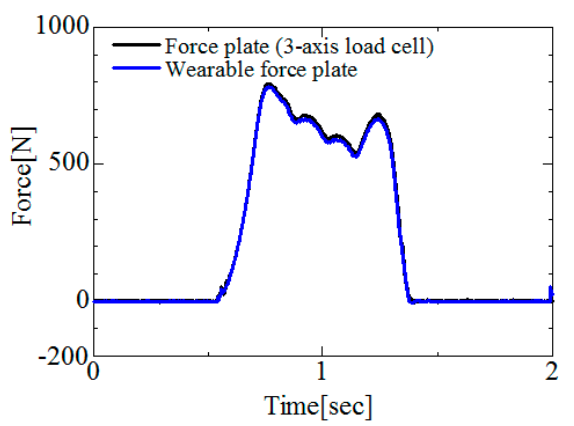

(c)

Figure 5. Results for force components on the force plate installed with 3-axis load cells and the wearable force plate; (a) $F_{x}$ (lateral direction) component; (b) $F_{y}$ (traveling direction) component; (c) $F_{z}$ (vertical direction) component.

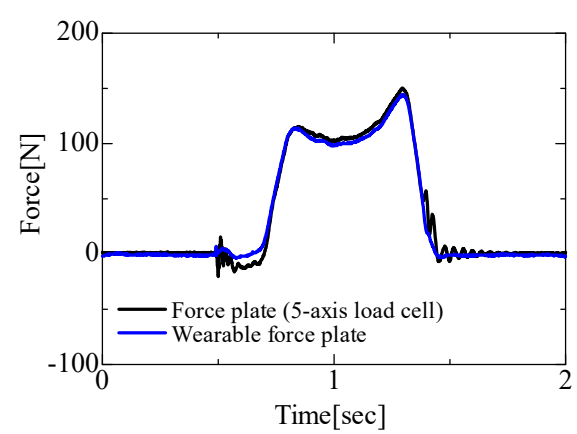

(a)

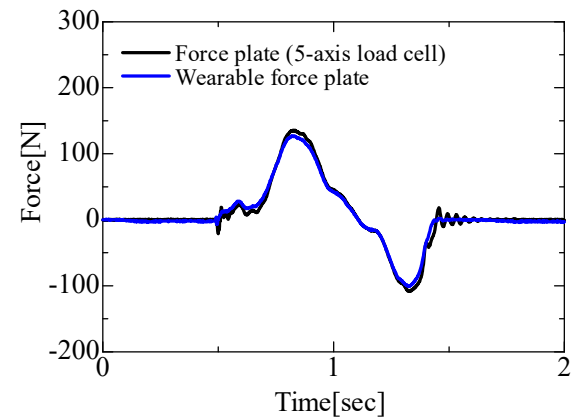

(b) 


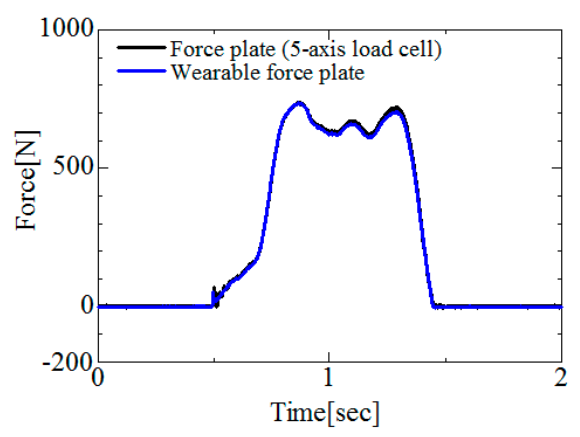

(c)

Figure 6. Results for force components on the force plate installed with 5-axis load cells and the wearable force plate; (a) $F_{x}$ (lateral direction) component; (b) $F_{y}$ (traveling direction) component; (c) $F_{z}$ (vertical direction) component.

\section{Conclusions}

In this study, we developed a new force plate system installed with five-axis load cells. The load cells measure a 3-axis force and a 2-axis moment. Therefore, this system can consider the effect of the torsion of top plate and compensation in measurement of outside parts of the load cells (Figure 3) using the moment components. The verification experiment using the force plates installed with the 3-axis load cells and the 5-axis load cells and the wearable force plate can indicate the performance of a developed force plate in the measurement between the force plates. The performance of the force plate is higher than the performance of a force plate installed with the 3-axis load cells. Therefore, the ground reaction force measurement of high performance in sports can be conducted using the developed force plate.

Funding: This research did not receive any specific grant from funding agencies in the public, commercial, or not-for-profit sectors.

Conflicts of Interest: The authors declare no conflict of interest.

\section{References}

1. Scorza: A.; Massaroni, C.; Orsini, C.; D'Anna, C.; Conforto, S.; Silvestri, S.; Sciuto, A. A Review on Methods and Devices for Force Platforms Calibration in Medical Applications. J. Eng. Sci. Technol. Rev. 2018, 11, 1018.

2. Kondo, A.; Doki, H.; Hirose, K. An Attempt for Measuring Snowboarding Performance Using Compact Force Sensors. Procedia Eng. 2012, 72, 86-91.

3. Adachi, W.; Tsujiuchi, N.; Koizumi, T.; Shiojima, K.; Tsuchiya, Y.; Inoue, Y. Development of Walking Analysis System Using by Motion Sensor with Mobile Force Plate. J. Syst. Des. Dyn. 2012, 6, 655-664.

4. Hirose, K; Doki, H.; Kondo, A. Studies on Orientation Measurement in Sports Using Inertial and Magnetic Field Sensors. J. Jpn. Soc. Sports Ind. 2012, 22, 255-262. (In Japanese)

(C) 2020 by the authors. Licensee MDPI, Basel, Switzerland. This article is an open access article distributed under the terms and conditions of the Creative Commons Attribution (CC BY) license (http://creativecommons.org/licenses/by/4.0/). 